Jan N. M. Schieveld

Judith A. van der Valk

Inge Smeets

Eline Berghmans

Renske Wassenberg

Piet L. M. N. Leroy

Gijs D. Vos

Jim van Os

\section{Diagnostic considerations regarding pediatric delirium: a review and a proposal for an algorithm for pediatric intensive care units}

Received: 5 March 2009

Accepted: 27 July 2009

Published online: 22 September 2009

(C) The Author(s) 2009. This article is published with open access at

Springerlink.com

J. N. M. Schieveld (®) •

I. Smeets - E. Berghmans · R. Wassenberg

Division of Child and Adolescent

Psychiatry, Department of Psychiatry and

Psychology, European Graduate School of

Neuroscience, SEARCH, Maastricht

University Medical Centre, PO Box 5800,

6202 AZ Maastricht, The Netherlands

e-mail: jan.schieveld@mumc.nl

J. A. van der Valk

Faculty of Health, Medicine and Life

Sciences, Maastricht University, Maastricht,

The Netherlands

P. L. M. N. Leroy · G. D. Vos

Division of Pediatric Intensive Care

Medicine, Department of Pediatrics,

Maastricht University Medical Centre,

PO Box 5800, 6202 AZ Maastricht,

The Netherlands
J. van Os

Department of Psychiatry and Psychology, European Graduate School of Neuroscience, SEARCH, Maastricht University Medical Centre, PO Box 616, 6202 AZ Maastricht, The Netherlands

J. van Os

Division of Psychological Medicine,

Institute of Psychiatry,

London SE5 8AF, UK

Abstract Context: If delirium is not diagnosed, it is unlikely that any effort will be made to reverse it.

Given evidence for under-diagnosis, tools that aid recognition are required. Objective: Relating three presentations of pediatric delirium (PD) to standard criteria and developing a diagnostic algorithm.

Results: Delirium-inducing factors, disturbance of consciousness and inattention are common in PICU patients: a pre-delirious state is present in most. An algorithm is introduced, containing (1) evaluation of the sedation-agitation level, (2) psychometric assessment of behavior and (3) opinion of the caregivers. Discussion: It may be argued that the behavioral focus of the algorithm would benefit from the inclusion of neurocognitive measures. Limitations: No sufficiently validated diagnostic instrument covering the entire algorithm is available yet. Conclusion: This is the first proposal for a PD diagnostic algorithm. Given the high prevalence of predelirious states at the PICU, daily evaluation is mandatory. Future algorithmic refinement is urgently required.

Keywords Delirium - Critical illness - Sickness behavior · Pediatric intensive care unit (PICU) . Diagnosis - Children · DSM-IV . RASS - PAED - CAM-ICU

\section{Introduction}

Delirium is a serious neuropsychiatric disorder. The Diagnostic and Statistical Manual of Mental Disorders (DSM-IV) characterizes delirium by four features: (1) inattention and disturbance of consciousness, (2) change in cognition, (3) acute onset and fluctuating course and (4) pathophysiological cause [1]. The prevalence in a general hospital is $10-30 \%$, rising to more than $50 \%$ in an intensive care unit (ICU) and around $80 \%$ in the terminally ill [2]. Research in adults indicates that delirium is associated with a worse functional outcome, longer hospital stay and higher mortality rate [2, 3]. Thus, systematic monitoring for delirium and appropriate treatment with haloperidol in critically ill adult patients were included in the 2002 clinical practice guidelines for sedatives and analgesia of the Society of Critical Care Medicine [4]. In oncologic and geriatric patients, delirium 
is often the single most important predictor of imminent death [2]. It is therefore vitally important to assess all critically ill patients on not five but six basic signs: pulse rate, temperature, respiratory rate, blood pressure, pain and mental status [5].

The most important step in delirium management is early recognition. The reason for this is that if delirium is not diagnosed, it is unlikely that any effort will be made to reverse it. Once delirium is detected, the first aim is to identify the underlying etiology. Often this can be achieved by a systematic assessment of the presence of known risk factors. Minimization and/or elimination of predisposing and precipitating factors thus is the natural main strategy in treating and preventing delirium. Management should focus on improving the patient's cognitive and emotional status and reduce the risk of adverse outcomes such as aspiration, prolonged immobility, increased length of acute care, institutionalization and death.

Despite its high incidence, medical staff has difficulties in diagnosing delirium. In 2008, Cheung and colleagues concluded that Canadian intensive care unit (ICU) intensivists diagnose delirium merely upon the presence or absence of an obvious medical etiology [6], and in a recent editorial regarding pediatric delirium in ICUs, this issue was referred to as "the big challenge" [7]. Diagnosing delirium in an ICU is a complex process, due to the presence of critical illness, high level of sedation and frequent mechanical ventilation, all of which complicate mental state assessment. There is some literature regarding diagnostic instruments in use for ICU delirium [6, 8]. The Confusion Assessment Method for the Intensive Care Unit (CAM-ICU) [9] and the Intensive Care Delirium Screening Checklist (ICDSC) [10], which were developed for adults in ICUs, are the most commonly used instruments in diagnosing delirium and are in good agreement [11].

\section{Pediatric delirium}

There is a dearth of literature regarding pediatric delirium, especially in relation to critical illness, which is likely due to unawareness of the clinical entity [8]. A PubMed search conducted on 22 June 2009 with the MESH headings " $p(a)$ ediatric delirium AND critical illness" yielded only six English language papers between 1993 and 2007. The DSM-IV section on child psychiatry [1] does not mention delirium, although the chapter "Delirium, Dementia, and Amnestic and Other Cognitive Disorders" devotes six lines to delirium in children. Only four major textbooks contain chapters or sections on pediatric delirium: "Handbook of Pediatric Emergencies" [12], "Child and Adolescence Psychiatry-A Comprehensive Textbook," 3rd edition [13], "Clinical Manual of
Pediatric Psychosomatic Medicine" [14, 15] and "Rutter's Child and Adolescent Psychiatry," 5th edition [15]. A recent study found that $33 \%$ of the PICU patients reported memories of psychotic features, including delusions and disturbing hallucinations, 3 months after discharge [16], suggestive of high rates of delirium. However, two studies in pediatric intensive care units (PICUs) found detection rates that did not exceed 5\% [17, $18]$.

There is no validated instrument for critically ill PICU patients. An instrument to conduct routine standardized assessments of mental status is urgently needed. Anecdotal evidence suggests that child psychiatrists are only consulted, if at all, when children present with agitation in the context of a hyperactive delirium. Hypoactive presentations, however, are also common, but often missed in the absence of active monitoring [19].

Apart from psychometric tools, there is a quest for delirium biomarkers. Candidate markers include hemoglobin-beta, S100B and IL-6; "however, the causal relationships remain to be investigated" [20]. The role of EEG in the diagnosis of delirium in critically ill adults is limited [21]. In children there are some case reports describing occipital EEG disturbances in PD, but there are no EEG studies focusing on PD in critical illness accompanied by mechanical ventilation. One study examined cytokines and EEG disturbances due to influenza and the relation with PD and/or febrile seizures in a non-PICU context-results, however, were essentially inconclusive [22].

Presently, there is no evidence that the treatment of PD improves outcome. However, given the aforementioned risks and sequelae in adults, the high level of discomfort and suffering in adult delirious patients, coupled with a lack of knowledge on these issues in critically ill children, it is necessary to reduce any risk and try to detect and treat PD as much as possible until proven otherwise.

The current article is intended as a guide in diagnosing delirium in PICU patients. First, the criteria of the DSMIV and the International Classification of Diseases and Related Health Problems (ICD-10) are discussed. Second, the main diagnostic instruments are reviewed, and third, an algorithm is introduced.

\section{Diagnostic considerations}

There are three manifestations of delirium of likely differential prognostic significance: pre-delirium (prodromal delirium), subsyndromal delirium and full-blown delirium $[23,24]$. These presentations have not yet been consistently and explicitly incorporated in current diagnostic classification systems [25]. The main diagnostic 
classification systems for delirium are the DSM-IV and the ICD-10, with respectively four and six criteria for delirium, which show a great degree of overlap $[1,26]$. The common criteria are disturbance of consciousness and inattention, cognitive changes, acute onset and fluctuating course, as well as a pathophysiological cause. The additional items of the ICD-10 are psychomotor and sleep-wake cycle disturbances.

Table 1 shows the relation among the four delirium criteria of the DSM-IV in relation to the three clinical presentations of delirium. In PICU patients with critical illness, a pathophysiological cause (criterion D) is always present. Frequently, there is also inattention and a disturbance of consciousness resulting from (1) the critical illness and the ensuing sickness behavior and (2) sedation. Sickness behavior is the behavioral repertoire of animals and humans that changes dramatically following disease, especially an infection. It includes reduced appetite, signs of fatigue, malaise and sleep disturbances, loss of interest in social activities, isolation, exaggerated responses to pain and failure to concentrate. These behavioral changes in mammals have evolutionary meaning: they promote recovery and protect the rest of the herd. Pro-inflammatory cytokines acting in the brain may cause sickness behavior [27-30]. Given the presence of sedation and sickness behavior in a PICU setting, resulting in inattention and a disturbance of consciousness, two of the four DSM-IV criteria are a priori positive (criterion D and A), resulting in a diagnosis of prodromal or pre-delirium. In other words, most patients in PICU settings are already pre-delirious. If three criteria are positive, the patient will be diagnosed as sub-syndromal. If all the criteria are positive, the diagnosis of delirium can be made.

Psychomotor disturbance is a criterion in ICD-10, but surprisingly not in DSM-IV. This feature is usually considered a core symptom of delirium and leads to the wellknown delirium classification into three types: hyperactive, hypoactive and mixed [31]. It is therefore proposed to initially evaluate both psychomotor disturbances and cognitive changes, as this will improve the diagnosis of delirium in a PICU context. We regard the sleep and sleep-wake cycle disturbance in a PICU patient - which is a criterion in ICD-10 but not in DSM-IV-as less relevant, because of the frequent use of sedation.
It can be questioned whether it is justified to use the DSM-IV criteria in young children. After all, children are not small adults: they have their own developmental stages and vulnerabilities. However, the clinical presentations of pediatric deliria are mostly equal to those in adults [8]. They have been described from the very early age of 16 months [32] and even 6 months [8, 33].

\section{Diagnostic instruments}

Several diagnostic tools for delirium exist, such as the Delirium Rating Scale (DRS) [34], the Delirium Rating Scale-Revised-98 (DRS-R-98) [35], the Pediatric Anesthesia Emergence Delirium Scale (PAED) [36], the CAMICU [9], and the ICDSC [10]. The DRS-88 and the DRSR-98 have been validated in adults $[34,35]$, but not in ICU settings. The PAED has been validated in children in the post-anesthesia phase in samples aged between 19 months and 6 years, but not in PICU patients [36]. The CAM-ICU and the ICDSC have not been validated in children, but have been studied in ICU settings [9, 10]. It is thought that the CAM-ICU and the ICDSC can be administered to children from the age of 8 years. The pediatric version of the CAM-ICU (p-CAM-ICU) is an age-adapted, easier version for children, which is now in its pilot phase [personal communication, Dr. Heidi Smith and Dr. Wes Ely, Vanderbilt University, Nashville, TN, October, 2008]. It is considered suitable in children from the age of 5 years. The p-CAM-ICU has not yet been validated; however, since the majority of PICU patients are aged under 3 years, varying from 50 to $80 \%$ depending on geographical location, the p-CAM-ICU will often not be suitable.

Many of the items of the above delirium instruments, particularly those concerning cognitive functions, are often not assessable in a PICU setting. This is due to the presence of critical illness, high levels of sedation, frequent use of mechanical ventilation, young age of the population and high proportion of patients with mental retardation. Therefore, it is important that a diagnostic tool for delirium in a PICU has its focus on behavioral (as in the PAED) rather than neurocognitive (as in the

Table 1 Three delirium presentations at the PICU in relation to the DSM-IV criteria

\begin{tabular}{lll}
\hline Number of DSM-IV criteria present & Which DSM-IV criteria are present? & Diagnosis \\
\hline 1 Criterion & Criterion D & No delirium \\
2 Criteria & Criterion D and A or B or C & Pre-delirium \\
3 Criteria & Criterion D and two of the following: A, B, C & Subsyndromal delirium \\
4 Criteria & All the criteria: A, B, C and D & Full-blown delirium \\
& & (hyperactive/hypoactive/mixed) \\
\hline
\end{tabular}

DSM-IV criteria: (A) inattention and disturbance of consciousness; (B) change in cognition; (C) acute onset and fluctuating course; (D) pathophysiological cause 
p-CAM-ICU) features, even though the behavioral features are often less conspicuous and therefore less reliable in diagnosing delirium when deep sedation is applied (with the exception of "fighting the ventilator" in a hyperactive delirium). Given these considerations, it is clear that the sedation-agitation level is an important part of the diagnostic evaluation of delirium in a PICU, requiring separate attention in a diagnostic algorithm.

\section{Diagnostic algorithm}

Given the complexity of the delirium concept and its context, a diagnostic algorithm was developed that may serve as a guide. The algorithm is the first part of a comprehensive flow chart comprising the various steps in the evaluation and management of the causes of emotional-behavioral disturbances at the PICU, which can vary from violent agitation in hyperactive delirium to the subtle complaint of the caregiver stating that "This is now no longer my child" in the hypoactive form. All available data sources may be used, including chart information.

The first step is the evaluation of the sedation-agitation level with the Richmond Agitation-Sedation Scale (RASS) [37], the second the psychometric assessment of behavior with the PAED scale and the evaluation of the opinion of the caregivers, the third the identification and management of somatic and pharmacological causes, the fourth and fifth the assessment and management of discomfort as well as assessment of possible moderating qualities of the psychosocial environment. The last step represents the treatment of delirium with medication. The two scales (RASS and PAED) are completed by the nursing staff.

Algorithm: Diagnostic algorithm for pediatric delirium at the PICU.

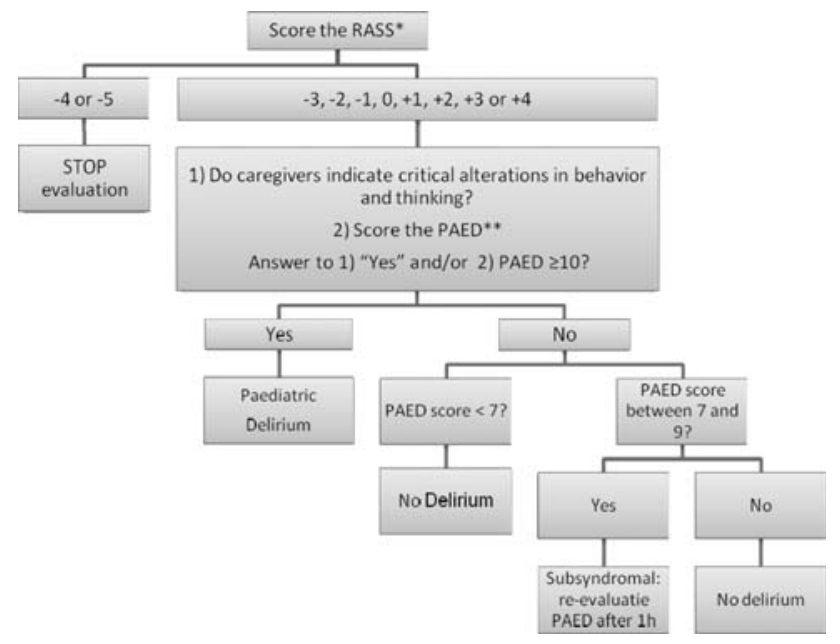

\section{*Procedure for RASS assessment.}

STEP 1 Observe patient. If patient is alert and calm, score 0 . If patient is restless or agitated, score +1 to $+4(+1=$ restless: anxious but movements not aggressive vigorous; $+2=$ agitated: frequent non-purposeful movement, fights ventilator; $+3=$ very agitated: pulls or removes tube(s) or catheter(s), is aggressive; $+4=$ combative: overtly combative, violent, immediate danger to staff).

STEP 2 If not alert, state patient's name and say to open eyes and look at speaker. If patient awakens with sustained eye opening and eye contact, score -1 ; if patient awakens with eye opening and eye contact, but not sustained, score -2 ; if patient has any movement in response to voice but no eye contact, score -3 .

STEP 3 When no response to verbal stimulation, physically stimulate patient by shaking shoulder and/ or rubbing sternum. If patient has any movement to physical stimulation, score -4 ; if patient has no response to any stimulation, score -5 .

\section{**Procedure for PAED assessment.}

Items:

1. The child makes eye contact with the caregiver.

2. The child's actions are purposeful.

3 . The child is aware of his/her surroundings.

4. The child is restless.

5. The child is inconsolable.

Observe the patient and score the items.

Items 1,2 and 3 are reversed scored as follows: $4=$ not at all; $3=$ just a little; $2=$ quite a bit; $1=$ very much; $0=$ extremely. Items 4 and 5 are scored as follows: $0=$ not at all; $1=$ just a little; $2=$ quite a bit; $3=$ very much; $4=$ extremely.

The scores of each item were summed to obtain a total Pediatric Anesthesia Emergence Delirium (PAED) scale score.

The algorithm is initiated by completing the RASS [37]. Scoring takes $20 \mathrm{~s}$. The RASS is a 10-point rating scale with four levels for agitation, five for sedation and one for calm, awake patients. Ratings are anchored according to a patient's responses to verbal and then to physical stimulation. The RASS forms the starting point of the algorithm, as evaluating consciousness is always a first step in a neuropsychiatric examination. Second, it is important to start the evaluation of mental status with an objective rating that may identify both hyperactive and hypoactive presentations.

The next step is the PAED and the opinion of the caregivers about critical alterations in behavior and/or thinking of the child. Clinical anecdotal evidence 


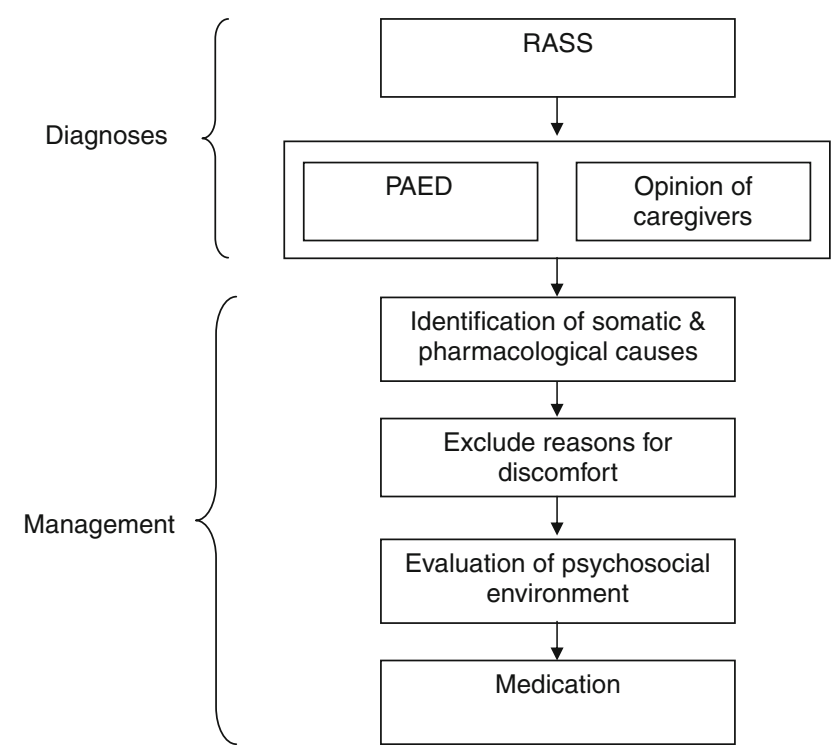

Fig. 1 Evaluation and management of emotional-behavioral disturbances at the PICU

indicates that their opinion is very important at least as an entry cue; it may even be considered diagnostic provided other causes have been excluded [see flow chart (Fig. 1)] in suspecting and diagnosing delirium [38]. Some caregiver observations will coincide with items of the PAED discussed below. Other observations, however, are: loss of acquired skills, regression, catatonic features and paranoid thinking $[15,39,40]$.

The PAED is an easy tool, with no a priori exclusion criteria, which measures behavioral features that reflect disturbance of consciousness, inattention, emotional and cognitive changes and psychomotor disturbances [36]. Since its introduction in 2004, six papers have been published regarding its value in children aged 1 to 6 years after an MRI procedure or anesthesiology for eye or dental surgery [41-46].

Rating takes $1 \mathrm{~min}$, and only minimal training is required. It is a 5-point scale with clear anchors. A score of $0-6$ suggests that no further evaluation is required. A score of 7-9 indicates that the patient may be subsyndromal; it is therefore very important to re-evaluate clinical state after $1 \mathrm{~h}$. A score $\geq 10$ is compatible with delirium. Our first results with the PAED in a PICU context are promising: in a pilot multidisciplinary observational study at the PICU $(n=139)$, children with PD scored significantly higher on PAED than non-delirious children: mean PAED total score for delirious children was 12.8 (SD 3.7) and 3.2 (SD $3.5)$ for non-delirious children. The optimal PAED cutoff value had a sensitivity of $81 \%$, a specificity of $91 \%$, a diagnostic likelihood ratio of 9.3 and a post-test probability of $97 \%$ (article in preparation).

Because of the fluctuating course, we recommend assessment with the RASS, as an entry cue for this algorithm, every hour, whereas the PAED should be completed every nursing shift, in order to miss as few cases of delirium as possible.

\section{Discussion}

To our knowledge, this is the first attempt towards a systematic and comprehensive approach in the evaluation and management of emotional and behavioral disturbances at the PICU, resulting in an algorithm for diagnosing pediatric delirium in critically ill children. One other research algorithm exists; this was developed for an adult ICU context consisting of two steps: (1) the CAM-ICU, and if not possible, (2) a chart review. This algorithm produced fair results [47]. For children, the algorithm presented in this paper is more suitable as it is child-specific.

It is doubtful whether there will ever be a good diagnostic instrument for delirium in the very youngest of PICU patients. The concept of "disorganized thinking" (a core feature of delirium) in a very young child is difficult to assess, because mental processes in a child's very early years are frequently either not verbally assessable or they are "unripe, illogical, magical" and thus, in the adult sense, disorganized. In our opinion, therefore, an observational instrument that evaluates behavioral changes-as a sign of acute brain failure (i.e., delirium) - has more value. In future research, we will try to lower the age range in which delirium can be diagnosed in a critically ill PICU patient with the use of a validated diagnostic instrument.

\section{Limitations}

Limitations of this algorithm are lack of empirical evidence and insufficient validation of the instruments. The algorithm does, however, provide the opportunity for further evaluation of the effectiveness of a systematic approach for PD at the PICU. All of the instruments have been validated, but none specifically in PICU patients aged between 0 and 18 years. The PAED has been validated only for children aged between 19 months and 6 years, and has not been validated in PICU settings. The RASS has not yet been validated in children but has been validated in adults in ICUs. Nevertheless, clinical experience suggests that this algorithm can still be very useful in PICUs. Research has been initiated to further validate the instruments and the algorithm.

Second, the algorithm cannot be carried out if patients are in a state of stupor or coma. Prior research in the elderly showed that in $85 \%$ of cases there is a transition from coma or stupor to delirium [48]. Therefore, it is important to be very attentive to this group. 
Acknowledgments We gratefully acknowledge the collaboration with and input from the colleagues from the Dutch consultationliaison child psychiatry work group: AnnePauline Cohen, Jeanne van Weel, Annelou de Vries, Monique van Lier, Elisabeth de Graeff- Meeder, Babette van Beusekom, Mijnke Janssen, Evelien Verbist, Miranda Fredriks, Fleur Wetsteijn, Ingrid MekkingPompen, Arjan Theil (chair) and Lourens J Kalverdijk, all child and adolescent psychiatrists; and of Laurens Voogd, MSc, and Jeanne Fincken, MSc, both senior medical students, and Jolien Nivelle, medical secretary.
Open Access This article is distributed under the terms of the Creative Commons Attribution Noncommercial License which permits any noncommercial use, distribution, and reproduction in any medium, provided the original author(s) and source are credited.

\section{References}

1. American Psychiatric Association (2000) Diagnostic and statistical manual of mental disorders, 4th edn text, revision (DSM-IV-TR). American Psychiatric Association, Washington DC

2. American Psychiatric Association (1999) Practice guidelines for the treatment of patients with delirium. Am J Psychiatr 5:1-20

3. Ouimet S, Kavanagh BP, Gottfried SB, Skrobik Y (2007) Incidence, risk factors and consequences of ICU delirium. Intens Care Med 33:66-73

4. Jacobi J, Fraser GL, Coursin DB, Riker RR, Fontaine D, Wittbrodt ET, Chalfin DB, Masica MF, Bjerke HS, Coplin WM, Crippen DW, Fuchs BD, Kelleher RM, Marik PE, Nasraway Jr SA, Murray MJ, Peruzzi WT, Lumb PD (2002) Clinical practise guidelines for the sustained use of sedatives and analgesics in the critically ill adult. Crit Care Med 30(1):119-141

5. Flaherty JH, Rudolph J, Shay K, Kamholz B, Boockvar KS,

Shaughnessy M, Shapiro R, Stein J, Weir C, Edes T (2007) Delirium is a serious and under-recognized problem: why assessment of mental status should be the sixth vital sign. J Am Med Dir Assoc 8:273-275

6. Cheung CZ, Alibhai SM, Robinson M, Tomlinson G, Chittock D, Drover J, Skrobik Y (2008) Recognition and labeling of delirium symptoms by intensivists: does it matter? Intens Care Med 34:437-446

7. de Carvalho WB, Fonseca MC (2008) Pediatric delirium: a new diagnostic challenge of which to be aware. Crit Care Med 36:1986-1987

8. Schieveld JNM (2008) On pediatric delirium in critical illness. A clinical multidisciplinary study in childneuropsychiatry at the PICU. Ph.D. thesis, Maastricht University, Maastricht. http://dissertaties.ub. unimaas.nl
9. Ely EW, Margolin R, Francis J, May L, Truman B, Dittus R, Speroff T, Gautam S, Bernard GR, Inouye SK (2001) Evaluation of delirium in critically ill patients: validation of the Confusion Assessment Method for the Intensive Care Unit (CAM-ICU). Crit Care Med 29:1370-1379

10. Bergeron N, Dubois MJ, Dumont M, Dial S, Skrobik Y (2001) Intensive care delirium screening checklist: evaluation of a new screening tool. Intens Care Med 27:859-864

11. Plaschke K, von Haken R, Scholz M, Engelhardt R, Brobeil A, Martin E, Weigand MA (2008) Comparison of the confusion assessment method for the intensive care unit (CAM-ICU) with the Intensive Care Delirium Screening Checklist (ICDSC) for delirium in critical care patients gives high agreement rate(s). Intens Care Med 34:431-436

12. Baldwin GA (2001) Handbook of pediatric emergencies. Lippincott, Williams \& Wilkins

13. Lewis M (2002) Child and adolescence psychiatry-a comprehensive textbook. Lippincott, William \& Wilkins

14. Shaw RJ, DeMaso DR (2006) Clinical Manual of Pediatric Psychosomatic Medicine-Mental Health Consultation With Physically Ill Children and Adolescents American Psychiatric Publishing, Inc

15. Rutter M, Bishop D, Pine D, Scott S, Stevenson J, Taylor E, Thapar A (2008) Rutter's child and adolescent psychiatry. Blackwell Publishing, Oxford

16. Colville G, Kerry S, Pierce C (2008) Children's factual and delusional memories of intensive care. Am J Respir Crit Care Med 177:976-982

17. Schieveld JN, Leroy PL, van Os J, Nicolai J, Vos GD, Leentjens AF (2007) Pediatric delirium in critical illness: phenomenology, clinical correlates and treatment response in 40 cases in the pediatric intensive care unit. Intens Care Med 33:1033-1040
18. Larsen GY, Donaldson AE, Parker HB, Grant MJ (2007) Preventable harm occurring to critically ill children. Pediatr Crit Care Med 8:331-336

19. Pandharipande P, Cotton BA, Shintani A, Thompson J, Costabile S, Truman Pun B, Dittus R, Ely EW (2007) Motoric subtypes of delirium in mechanically ventilated surgical and trauma intensive care unit patients. Intens Care Med 33:1726-1731

20. van Munster BC (2009) Pathophysiological studies in delirium, a focus on genetics. Ph.D thesis, University of Amsterdam

21. Ely EW, Truman B, Manzi DJ, Sigl JC, Shintani A, Bernard GR (2004) Consciousness monitoring in ventilated patients: bispectral EEG monitors arousal not delirium. Intens Care Med 30:1537-1543

22. Fukumoto Y, Okumura A, Hayakawa F, Suzuki M, Kato T, Watanabe K, Morishima T (2007) Serum levels of cytokines and EEG findings in children with influenza associated with mild neurological complications. Brain Dev 29:425-430

23. Ouimet S, Riker RR, Bergeron N, Cossette M, Kavanagh BP, Skrobik YK (2007) Subsyndromal delirium in the ICU: evidence for a disease spectrum. Intens Care Med 33:1007-1013

24. McCusker J, Cole MG, Dendukuri N, Belzile E (2003) Does delirium increase hospital stay? J Am Geriatr Soc 51:1539-1546

25. Cole MG, McCusker J, Ciampi A, Belzile E (2008) The 6- and 12-month outcomes of older medical inpatients who recover from subsyndromal delirium. J Am Geriatr Soc 56:2093-2099

26. World Health Organisation (2004) ICD-10: International classification of diseases and related health problems: 10th revision. World Health Organisation, Geneva 
27. Dantzer R, Konsman JP, Bluthe RM, Kelley KW (2000) Neural and humoral pathways of communication from the immune system to the brain: parallel or convergent? Auton Neurosci 85:60-65

28. Kelley KW, Bluthe RM, Dantzer R, Zhou JH, Shen WH, Johnson RW, Broussard SR (2003) Cytokine-induced sickness behavior. Brain Behav Immun 17:112-118

29. Teeling JL, Felton LM, Deacon RMJ, Cunningham C, Rawlins JNP, Perry VH (2007) Sub-pyrogenic systemic inflammation impacts on brain and behavior, independent of cytokines. Brain Behav Immun 21:836-850

30. Maclullich AMJ, Ferguson KJ, Miller T, de Rooij SEJA, Cunningham C (2008) Unravelling the pathophysiology of delirium: a focus on the role of aberrant stress responses. J Psychosom Res 65:229-238

31. Trzepacz PT, Meagher DJ (2005) Delirium. In: Levinson JL (ed) Textbook of psychosomatic medicine. The American Psychiatric Publishing, Washington DC, pp 92-130

32. Kanner L (1935) Child psychiatry. Chas. C. Thomas, Springfield

33. Turkel SB, Tavare CJ (2003) Delirium in children and adolescents. $\mathbf{J}$ Neuropsych Clin Neurosci 15:431-435

34. Trzepacz PT, Baker RW, Greenhouse J (1988) A symptom rating scale for delirium. Psychiatr Res 23:89-97

35. Trzepacz PT, Mittal D, Torres R, Kanary K, Norton J, Jimerson N (2001) Validation of the Delirium Rating Scale-revised-98: comparison with the delirium rating scale and the cognitive test for delirium. J Neuropsychiatr Clin Neurosci 13:229-242
36. Sikich N, Lerman J (2004)

Development and psychometric evaluation of the pediatric anesthesia emergence delirium scale. Anesthesiology 100:1138-1145

37. Ely EW, Truman B, Shintani A, Thomason JW, Wheeler AP, Gordon S, Francis J, Speroff T, Gautam S, Margolin R, Sessler CN, Dittus RS, Bernard GR (2003) Monitoring sedation status over time in ICU patients: reliability and validity of the Richmond Agitation-Sedation Scale (RASS). Jama 289:2983-2991

38. Stoddard FJ, Wilens TE (1995) Delirium. In: Jellinek MS, Herzog DB (eds) Psychiatric aspects of general hospital pediatrics. Yearbook Medical Publishers, Chicago, pp 254-259

39. Schieveld JN, Leentjens AF (2005) Delirium in severely ill young children in the pediatric intensive care unit (PICU). J Am Acad Child Adolesc Psychiatr 44:392-394 discussion 395

40. Stoddard FJ, Wilens TE (1995) Delirium. In: Jellinek MS, Herzog DB (eds) Psychiatric aspects of general hospital pediatrics. Chicago

41. Isik B, Arslan M, Tunga AD, Kurtipek O (2006) Dexmedetomidine decreases emergence agitation in pediatric patients after sevoflurane anesthesia without surgery. Paediatr Anaesth 16:748-753

42. Abu-Shahwan I, Chowdary K (2007) Ketamine is effective in decreasing the incidence of emergence agitation in children undergoing dental repair under sevoflurane general anesthesia. Paediatr Anaesth 17:846-850
43. Aouad MT, Yazbeck-Karam VG, Nasr VG, El-Khatib MF, Kanazi GE, Bleik JH (2007) A single dose of propofol at the end of surgery for the prevention of emergence agitation in children undergoing strabismus surgery during sevoflurane anesthesia. Anesthesiology 107:733-738

44. Abu-Shahwan I (2008) Effect of propofol on emergence behavior in children after sevoflurane general anesthesia. Paediatr Anaesth 18:55-59

45. Ozdemir Kol I, Egilmez H, Kaygusuz K, Gursoy S, Mimaroglu C (2008) Open-label, prospective, randomized comparison of propofol and sevoflurane for laryngeal mask anesthesia for magnetic resonance imaging in pediatric patients. Clin Ther 30:175-181

46. Hasani A, Ozgen S, Baftiu N (2009) Emergence agitation in children after propofol versus halothane anesthesia. Med Sci Monit 15:CR302-CR306

47. Pisani MA, Araujo KL, Van Ness PH, Zhang Y, Ely EW, Inouye SK (2006) A research algorithm to improve detection of delirium in the intensive care unit. Crit Care 10:R121

48. McNicoll L, Pisani MA, Zhang Y, Ely EW, Siegel MD, Inouye SK (2003) Delirium in the intensive care unit: occurrence and clinical course in older patients. J Am Geriatr Soc 51:591-598 